\title{
REMOVAL OF NAPHTHALENE FROM AQUEOUS SOLUTION ON CHEMICALLY MODIFIED ACTIVATED CARBONS
}

CO Ania, B Cabal, C Pevida, A Arenillas, JB Parra ${ }^{*}$, F Rubiera, JJ Pis

Instituto Nacional del Carbón, CSIC. Apartado 73, 33080 Oviedo (Spain)

${ }^{*}$ Corresponding author

JB Parra, Instituto Nacional del Carbon, CSIC, P.O. Box 73, 33080 Oviedo, Spain e-mail: jbparra@incar.csic.es

\begin{abstract}
The aim of this work was to correlate the textural and chemical features of carbonaceous adsorbents with the adsorption capacity of naphthalene from aqueous phase, at the concentration in which this compound is usually found in wastewater from coke ovens. The study reveals that the adsorption capacity in different carbon materials depends not only on the textural characteristics of the material but also on the functionalities of the activated carbons. The micropores of the adsorbents, particularly those of narrower diameter, were found to be active sites for the retention of naphthalene. In contrast, the modification of the surface chemistry of the carbon materials led to a decrease in the adsorption capacities. Dispersive forces play an important role, and adsorbents with a higher non-polar character have proven to be more efficient for the naphthalene adsorption. This behaviour has been linked to the presence of specific interactions between the basal planes and the polyaromatic structure of the naphthalene molecule.
\end{abstract}

Keywords: PAH, adsorption, liquid phase, activated carbon 


\section{Introduction}

The coking process involves the production of coke from the pyrolysis of suitable grades of coal and the processing of coke oven gas to remove major pollutants such as tar, ammonia, light oils, and sulphur compounds, before the gas is used as fuel for heating the ovens. Although the coke making industry has improved the technique over the years, it is still a major source of fugitive and stack emissions of toxic compounds to the atmosphere and water streams (World Bank Group, 1998). Pollution prevention in coke making has been mainly focused on reducing air emissions and the implementation of cleaner production processes, while few studies are reported on the minimization of pollutants from wastewater (Seredych and Gierak, 2004; Seredych et al., 2005). In this regard, the production-related targets consider that the generation of wastewater should be less than $0.3 \mathrm{~m}^{3} / \mathrm{t}$ of coke.

The coking process nowadays generates typically between 0.4 and $4 \mathrm{~m}^{3}$ of wastewater per tonne of coke, containing a wide variety of pollutants in extremely high concentrations (i.e., phenols, cyanides, thiocyanate, hydrocarbons). Wastewaters also contain PAHs at significant concentrations (up to $30 \mathrm{mg} \mathrm{L}^{-1}$ ), which produce an effluent with a high COD (chemical oxygen demand) and a low BOD (biological oxygen demand). These compounds are considered as priority pollutants (Directive 2000/60/EC; Directive 96/62/EC) as they are long-term persistent and highly mobile throughout the environment (semi-volatiles). Most of them also present carcinogenic properties.

Between the conventional methods for purification of wastewater, biological treatment is by far the most widely applied and cost effective one. Unfortunately, due to the low biodegradability of some PAHs, these compounds cannot be easily destroyed by biological treatment and, in addition, many of them can inhibit the biological process. 
Activated carbon, however, is able to deal with almost any organic compound present in the waste. Some advantages such as the low-energy demanding process, the possibility of regenerating the spent adsorbent, the broad availability of adsorbents and the adsorption of PAH from liquid phase on activated carbons, make this an interesting field of research.

Until now, extensive research has been carried out on the adsorption of organic compounds from dilute aqueous solutions by activated carbons. In particular, the adsorption of phenol and related compounds is one of the most studied systems in liquid-phase applications of carbon adsorbents, due to their industrial and environmental importance (Terzyk, 2003; Leon-Leon and Radovic, 1993; Moreno-Castilla et al., 1995; Ania et al., 2004). Nevertheless, there is still some lack of knowledge of the relationship between the active sites on the adsorbent and the later adsorptive capacities of organic compounds in the aqueous media. Moreover, most of the research into pollution by PAH has been performed in the gas phase (Mastral et al., 2003), while investigation on aqueous streams has remained a little behind.

The main objective of this research is to explore the performance of carbon adsorbents as a media for naphthalene removal, one of the most abundant polyaromatic compounds in wastewater from the coke making industry. Special attention was paid to the many factors that affect the competitive and complex process of adsorption from diluted solutions. The effect of the surface functionalities on carbons was investigated by carefully modifying the surface chemistry of the carbon materials by wet oxidation. A series of adsorbents with a similar porous structure but different surface chemistry were prepared. A proper understanding of the role of the activated carbon surface chemistry in the retention of $\mathrm{PAH}$ is critical for the selection and production of adsorbents for the removal of PAH from an aqueous medium. 


\section{Materials and methods}

\subsection{Materials}

A commercial activated carbon -B-, obtained from physical activation of coal and supplied by Agrovin S.A. was chosen for this study. This adsorbent is commonly used for the purification of industrial wastewater and drinking water. The samples were ground, sieved and a particle size fraction of $0.71-1 \mathrm{~mm}$ was used in all the experiments. A series of activated carbons with a similar porous structure but different surface chemistry was prepared by wet oxidation with nitric acid at different concentrations (20 and $60 \%$ ) and ammonium persulfate as described elsewhere (Ania et al., 2004). The samples will be referred to in the text as BN20, BN60 and BS, respectively. After oxidation, the samples were washed and dried at $110{ }^{\circ} \mathrm{C}$ overnight. The ultimate analyses of the carbons are summarized in Table 1.

\subsection{Adsorption from solution}

Adsorption measurements of naphthalene from aqueous solutions on carbon adsorbents were performed at room temperature in a stirred batch system, thermostatically controlled with an external circulating bath. Kinetic studies revealed that the adsorption equilibrium was established after 3 days. Details of the procedure followed for the measurement of the adsorption isotherms are described elsewhere (Ania et al., 2004). Briefly, different amounts of carbons (from $50 \mathrm{mg}$ to $1 \mathrm{~g}$ ) were weighed and added to flasks containing $0.1 \mathrm{~L}$ of a naphthalene solution of concentration $30 \mathrm{mg} \mathrm{L}^{-1}$. The covered flasks were shaken for $72 \mathrm{~h}$ at a constant temperature. Initial and equilibrium concentrations of the aqueous solutions were measured using a UV spectrometer at the wavelength of $275.5 \mathrm{~nm}$. 
The equilibrium data were fitted to the so-called Langmuir-Freundlich (LF) single solute isotherm (Derylo-Marczewska et al., 1984), which has the equation:

$\vartheta_{t}=\frac{q_{e}}{q_{o}}=\frac{(K C)^{n}}{1+(K C)^{n}}$

where $q_{e}$ is the adsorbed amount of solute per unit gram of adsorbent, $q_{o}$ is its maximum adsorption per unit mass of the adsorbent, $K$ is the Langmuir-type constant defined by the van't Hoff equation, and the exponential term $n$ represents the heterogeneity of the site energies.

\subsection{Textural and chemical characterization}

Textural characterization was carried out by measuring the $\mathrm{N}_{2}$ adsorption isotherms at $-196^{\circ} \mathrm{C}$ in an automatic apparatus (Micromeritics ASAP 2010M). Before the experiments, the samples were outgassed under vacuum at $120{ }^{\circ} \mathrm{C}$ overnight. The isotherms were used to calculate the specific surface area, $\mathrm{S}_{\mathrm{BET}}$, total pore volume, $\mathrm{V}_{\mathrm{T}}$, and pore size distributions. The pore size distributions were evaluated using the density functional theory (DFT) (Olivier, 1995). Additionally, the distribution of pores smaller than $0.7 \mathrm{~nm}$ (narrow microporosity) was assessed from $\mathrm{CO}_{2}$ adsorption isotherms at $0{ }^{\circ} \mathrm{C}$ (Lozano-Castello et al., 2004) performed in a Micromeritics Gemini 2375 with DR formulism, using $1.023 \mathrm{~g} \mathrm{~cm}^{-3}$ as the density of adsorbed $\mathrm{CO}_{2}$ and 0.36 as $\beta$ parameter.

\subsection{Thermal analysis}

Thermal analysis was carried out using a TGA92 thermogravimetric analyzer from Setaram (TG). Temperature programmed desorption (TPD) experiments were carried out under an argon flow rate of $50 \mathrm{~cm}^{3} \mathrm{~min}^{-1}$, at a heating rate of $15^{\circ} \mathrm{C} \mathrm{min}{ }^{-1}$, up to a final temperature of $1000{ }^{\circ} \mathrm{C}$. An Omnistar quadrupole mass spectrometer from Balzers 
(MS), and a Nexus infrared spectrometer from Nicolet (FTIR), linked to the thermobalance were used for evolved gas analysis. To avoid secondary reactions, a probe was placed very close to the sample crucible of the thermobalance in the direction of the gas flow. The gas lines between the TG and the MS/FTIR were heated to $180^{\circ} \mathrm{C}$ in order to avoid cold points. For each experiment, about $35 \mathrm{mg}$ of a ground carbon sample was used. In this way, additional information regarding the identification of the decomposition products was obtained, along with the evolution of the rate of mass loss with temperature for the samples studied.

\section{Results and discussion}

\subsection{Textural properties and chemical composition of the as-received samples}

Detailed characteristics of the pore structure of the series of modified carbons are presented in Table 2. The nitrogen adsorption isotherms are shown in Figure 1. It can be observed that all the isotherms belong to type I of the BDDT classification, which indicates that carbons, even after modification, remain mainly microporous. The oxidized samples BS and BN20 showed a slight decrease in nitrogen uptake, as compared to the initial counterpart, indicating minor modification in the pore volume and pore sizes. This effect was much greater in the case of $\mathrm{BN} 60$. The $\mathrm{N}_{2}$ adsorption isotherms also show a H4 type hysteresis loop, characteristic of the slit-shaped pores where the adsorption and desorption branches are parallel (Arnold, 1995).

Analysis of the data obtained from the nitrogen adsorption isotherms indicates that upon oxidation treatment, the porous features of the adsorbents were not much altered, except in the case of BN60. Oxidation in mild conditions (i.e., persulfate and $20 \%$ nitric acid) showed a slight decrease in nitrogen uptake, as compared to the initial counterpart, indicating minor modification in the pore volume and pore sizes. The apparent surface areas of these samples decreased by 9.3 and $13.2 \%$, respectively, whereas the volume 
of total micropores accessible to the nitrogen probe decreased by 13 and $18 \%$, respectively. By contrast, this decrease reached $27 \%$ in the sample treated under the most aggressive conditions of concentrated nitric acid (BN60).

An exhaustive analysis of the pore size distributions (PSD) combining the information of $\mathrm{N}_{2}$ and $\mathrm{CO}_{2}$ adsorption isotherms, indicated that the changes induced in the samples affected both the micro and mesoporosity (Table 2). The narrow microporosity evaluated from the $\mathrm{CO}_{2}$ data increased in the case of BS and BN20, suggesting an effect of opening porosity, while it remained almost constant in BN60. In contrast, the volume of total micropores determined by nitrogen decreased. These results revealed that the alterations in microporosity are mostly created in the micropores of larger sizes.

The changes in surface chemistry after oxidation and the nature of the functionalities created were studied by different techniques (i.e., TPD-MS/FTIR, (point of zero charge -PZC- and chemical analysis) and the results are summarized in Table 1. The TPD results include the quantification of the $\mathrm{CO}$ and $\mathrm{CO}_{2}$ evolved as temperature increases in an argon atmosphere. This supplies information on the surface chemistry of the carbon material. The $\mathrm{CO}_{2}$ evolves at low temperatures as a result of the decomposition of surface groups of an acid nature, whereas the $\mathrm{CO}$ comes from weakly acidic, neutral and basic groups, which are more thermally stable and therefore evolve at higher temperatures (Haydar et al., 2000).

As expected, the oxidized samples become more acidic (lower PZC values) indicating that the majority of the functionalities created are of an acidic nature. It should be underlined that BS presented an almost 1.5 times smaller oxygen content than BN60, despite their similar PZC values. This is indicative of differences between the nature of the surface oxygen groups created in samples BS and BN60. These results were corroborated by the great difference between the amounts of $\mathrm{CO}_{2}$ and $\mathrm{CO}$ desorbed 
during the TPD experiments. That is, oxidation in mild conditions (i.e., ammonium persulphate) created groups of a stronger acidic character in the carbon (i.e, carboxylic acids) whereas the use of concentrated nitric acid gave rise to larger amounts of functionalities (possible phenolic groups) of a weaker acidic nature.

In sum, it can be clearly seen that, as a result of the oxidation using both oxidizing agents, the porous texture did not change considerably, although it is clear that the higher $\mathrm{HNO}_{3}$ concentration $(60 \%)$ produced some decrease in porosity (Table 2). Hence, it is possible to carefully modify the surface chemistry of an activated carbon in order to prepare a series of adsorbents with a similar porous structure but different surface chemistry. In this way, a proper understanding of the role of activated carbon surface chemistry in the retention of naphthalene from aqueous medium can be acquired.

\subsection{Adsorption of naphthalene from liquid phase}

Figure 2 illustrates the naphthalene adsorption isotherms on the carbons. All of them belong to the L type in the Giles classification (Giles et al., 1960). Therefore, no strong competition of the solvent takes place for the active sites of adsorption. The adsorption isotherms show a tendency to reach a plateau at a high equilibrium concentration of naphthalene (i.e., low dose of adsorbent). Since the amount adsorbed steadily increases, the saturation limit at low adsorbent doses is not attained. The adsorptive capacity of the carbons was obtained by fitting the experimental data to the LF approach. The results are shown in Table 3, along with the LF parameters of surface heterogeneity and the linear range and correlation coefficients. The excellent fitting indicates that the LF equation is suitable for application for the systems studied. According to the results observed, the adsorption capacity decreased in the modified carbons, even when the changes in the pore structure were somewhat smaller. From Table 3 and Figure 2, it can 
be concluded that the adsorption capacity is higher for those activated carbons with a low concentration of surface oxygen groups, i.e. B sample, and lower for those activated carbons with an increasing concentration of surface oxygen groups. These results contrast with those reported by other authors (Seredych and Gierak, 2004; Seredych et al., 2005) which showed a positive enhancement of naphthalene uptake on synthetic carbons oxidized with nitric acid 6M. In such case, this behavior might likely be related to the pore size of the oxidized carbon, notwithstanding its theoretical surface chemistry which was not thereby reported.

Furthermore, it is well known that the micropores are active sites in adsorption processes of organic compounds, both in gas or liquid phase (Ania and Bandosz, 2005; Ania et al., 2004; Garcia et al., 2004). Taking into account the small size of the naphthalene molecule -the estimated value of $7 \AA$ was calculated using MS Modelling software (Kao and Allinger, 1977)-, one would expect adsorption to be produced mainly in micropores of similar sizes (i.e, narrow microporosity) where the adsorption potential is higher. A close look at the isotherms shows that naphthalene adsorption does not follow this trend relating to the porosity. In fact, after oxidation in mild conditions there was an increase in the volume of narrow micropores (see Table 2). However, the carbons showed a sharp decrease in naphthalene retention. The results in Table 3 confirm that the differences in porosity cannot explain the adsorption capacities and state that the surface chemistry (and particularly the oxygen-containing created groups) must play an important role in naphthalene uptake. Thus, to investigate the role of the chemical nature of the carbons in the lower adsorption of naphthalene, the nature of the interactions between the adsorbate and the adsorbents has to be addressed.

DTG curves of the carbons after naphthalene exposure were analyzed (Figure 3). A common feature in all cases is a peak at temperatures lower than $100{ }^{\circ} \mathrm{C}$, due to the 
removal of physisorbed water. In order to avoid a misinterpretation of these results, it must be stressed that the peaks at temperatures higher than $700{ }^{\circ} \mathrm{C}$, which appeared in all the oxidized samples, are linked to the decomposition of the surface functionalities, since they are also observed in the profiles of the oxidized carbons before naphthalene adsorption.

For all the samples one peak of different width and intensity was observed after naphthalene adsorption, which was not detected before the uptake (Figure 3). This peak, centred at around $400^{\circ} \mathrm{C}$, is thus assigned to the removal of naphthalene physisorbed in the porous structure of the carbons. In the case of the oxidized carbons, there is an small overlap of this peak with that attributed to the decomposition of carboxylic groups, which is also present before naphthalene exposure (Figure 4). Nevertheless, after naphthalene adsorption the intensity of the peak increases, due to the naphthalene evolution. This fact is corroborated by the identification of the evolved gases by TGFTIR, as it will be discussed below.

The high temperature of this peak suggests that the adsorption takes place mainly in active sites of high adsorption potential; this is in pores whose dimensions match those of the adsorbate (i.e, narrow microporosity). It should be noted that this desorption peak appeared at almost the same temperature for all samples (except BN60), regardless of the type or amount of surface groups on the carbon surface, suggesting that the same forces of adsorption are involved in all cases. In the case of BN60, the peak shifted to lower temperatures, which is consistent with the detrimental effect on the microporosity of the carbon after treatment with concentrated nitric acid. The absence of a second peak at higher temperatures, observed for instance in the case of other organic molecules (i.e., phenol) (Ania et al., 2005), indicates a lack of specific interactions 
between the surface functionalities and the naphthalene molecule, which seems reasonable taking into account the non-polar nature of this compound.

When TG is coupled with evolved gas analysis, additional information about the desorbed species can be obtained. In this work, the gases evolved from the TPD experiments were analyzed by FTIR analyzer. The results showed the evolution of naphthalene at temperatures above $300^{\circ} \mathrm{C}$ (Figure 4). The fact that the naphthalene desorption peak detected by FTIR appears at temperatures higher than those shown by DTG curve is probably due to the condensation of this compound after it has evolved and before it has reached the infrared gas analysis cell. The gas lines from the TG to the MS and FTIR were kept at $160-180^{\circ} \mathrm{C}$, but since the boiling point of this compound is $220^{\circ} \mathrm{C}$, and the temperature of desorption was above $300^{\circ} \mathrm{C}$, cold points should be expected to appear in the system leading to the condensation of naphthalene. Nevertheless, on the basis of the TG-FTIR results it can be inferred that the process of naphthalene adsorption is a reversible one and that naphthalene does not seem to undergo decomposition reactions while heating. This finding is very important from the point of view of the regeneration of the adsorbent and will be further investigated in future studies.

Support for the role of narrow micropores in the adsorption of naphthalene was provided by the analysis of the porosity of the samples after adsorption (Table 2). For this purpose, $\mathrm{N}_{2}$ and $\mathrm{CO}_{2}$ adsorption isotherms were recorded for the samples loaded with naphthalene, after being washed with water to remove the excess of the adsorbate on the outer part of the carbon, and dried at $120^{\circ} \mathrm{C}$. Before the experiments, the samples were outgassed at $120^{\circ} \mathrm{C}$ under vacuum.

The apparent BET surface areas and pore volumes decreased between 26-50\% after adsorption. Regarding the PSD, both the micro and mesoporosity significantly changed 
when the samples were loaded with naphthalene. The main difference in the microporosity, as a result of naphthalene adsorption, is a decrease in the volumes of pores narrower than $1 \mathrm{~nm}$ (Figure 5). In addition, the volumes of mesopores are sharply reduced. Both effects were especially remarkable in the parent carbon -B- which has the lower oxygen content. The same trend can be observed from the $\mathrm{CO}_{2}$ adsorption isotherms shown in Figure 6, underlining the important role of narrow microporosity in naphthalene uptake. The highest decrease in $\mathrm{CO}_{2}$ uptake after naphthalene adsorption corresponds to the non-modified sample -B-, which is in good agreement with the largest adsorptive capacity for the sample achieved in liquid phase.

Moreover, as mentioned before, the changes in porosity cannot explain the behavior of the carbons in the uptake of naphthalene. Therefore, the challenge is to elucidate the exact role of the functionalities in the adsorption process of this aromatic hydrocarbon.

Although the results confirmed that the adsorption of naphthalene depends greatly on the pore size distribution of the adsorbent, dispersive forces are expected, taking into account the non-polar nature of both the adsorbate and the graphene layers of the carbons. Assuming a mechanism similar to the one reported in the case of aromatic compounds, like phenols and derivates (Leon-Leon and Radovic, 1993; Moreno-Castilla et al., 1995), naphthalene molecules may interact specifically with the carbon surface due to their polyaromatic structure, involving interactions of the electron-rich regions located in the graphene layers with the $\pi$ electrons of the adsorbate.

In this regard, it has to be taken into account that the functionalization of carbons mainly occurs on the edges of the carbon crystallites, since carbon atoms within the basal plane sites and surrounded by other carbon atoms exhibit a far lower reactivity towards oxidation than those located at the periphery of such segments -so-called edge sites- (Henning, 1966; Donnet, 1982). This will also affect the structure and chemical 
nature of the basal planes of the carbon. Hence, as a result of oxidation, the edges of the carbons are functionalized with electron-acceptor groups (ie., carboxylic and phenolic groups) and $\pi$ electron density is withdrawn from the graphene layers of the basal planes. As a result, the strength of the dispersive interactions decrease, and fewer naphthalene molecules cover the carbon surface. Then higher $\pi$-electron densities (arising from the low oxygen contents) result in a larger adsorption from liquid phase, as corroborated by the experimental data from liquid phase adsorption. At the same time, the functionalization of the carbon also increases the polar character of the adsorbent (Table 1), which is responsible for the negative effect on the dispersive forces of a non-polar nature.

\section{Conclusions}

The important effect of the surface chemistry of activated carbons on the adsorption of naphthalene from aqueous solution has been stated by evaluating adsorbents with a similar porosity and different surface chemistry.

The results confirmed that the adsorption of naphthalene strongly depends on the pore size distribution of the adsorbent, particularly microporosity. The study of the textural properties of the carbons after the adsorption of naphthalene revealed that the main difference in microporosity is a decrease in the volumes of pores narrower than $1 \mathrm{~nm}$. The same trend can be observed from the $\mathrm{CO}_{2}$ adsorption isotherms, underlining the important role of narrow microporosity in the uptake of naphthalene.

Adsorbents with a higher non-polar character (i.e., low oxygen content) have proven to be more efficient for naphthalene adsorption. This behaviour has been linked to the presence of dispersive interactions between the basal planes and the polyaromatic structure of the naphthalene molecule. 


\section{Acknowledgements}

Work carried out with a financial grant from the European Coal and Steel Community (Project 7220-PR139). We also thank Agrovin S.A. for kindly supplying the activated carbon. COA thanks the Spanish MEC for a postdoctoral fellowship (MEC EX20040612). 


\section{References}

Ania, C.O., Bandosz, T.J., 2005. Importance of structural and chemical heterogeneity of activated carbon surfaces for adsorption of dibenzothiophene. Langmuir 21, 77527759.

Ania, C.O., Parra, J.B., Pevida, C., Arenillas, A., Rubiera, F., Pis, J.J., 2005. Pyrolysis of activated carbons exhausted with organic compounds. J. Anal. Appl. Pyrol., 74, 518-524.

Ania, C.O., Parra, J.B., Pis, J.J., 2004. Oxygen-induced Decrease in the Equilibrium Adsorptive Capacities of Activated Carbons. Ads. Sci. Technol. 22, 337-351.

Arnold, E., 1995. In: Porosity in carbons. UK: Ed John W Patrick.

Derylo-Marczewska, A., Jaroniec, M., Gelbin, D., Seidel, A., 1984. Heterogeneity effects in single-solute adsorption from dilute solutions on solids. Chemica Scripta 24, 239-246.

Directive 2000/60/EC, EU Water Framework Directive. Official Journal L 327, on 22 December 2000.

Directive 96/62/EC, EU Air Quality Framework Directive. Official Journal L 309, on 27 November 2001.

Donnet, J.B., 1982. Structure and reactivity of carbons: from carbon black to carbon composites. Carbon 20, 267-282.

Garcia, T., Murillo, R., Cazorla-Amoros, D., Mastral, A.M., Linares-Solano, A., 2004. Role of the activated carbon surface chemistry in the adsorption of phenanthrene. Carbon 42, 1683-1689.

Giles, C., Mc Ewan, T., Nakhwa, S., Smith, D.J., 1960. Studies in Adsorption. Part XI. A system of classification of solutions adsorption isotherms, and its use in diagnosis 
of adsorption mechanisms and in measurement of specific surface areas of solids. J. Chem. Soc., 3973.

Haydar, S., Moreno-Castilla, C., Ferro-Garcia, M.A., Carrasco-Marin, F., RiveraUtrilla, J., Perrard, A., Joly, J.P., 2000. Regularities in the temperature-programmed desorption spectra of $\mathrm{CO}_{2}$ and $\mathrm{CO}$ from activated carbons. Carbon 38, 1297-1308.

Henning, G.R., 1966. Electron Microscopy of reactivity changes near lattice defects in graphite. In: Chemistry and Physics of Carbon, (P.L. Walker, Jr., Ed.), Vol. 2, Ch. 1, pp. 1-49.

Kao, J., Allinger, N.L., 1977. Conformational-analysis.122. Heats of formation of conjugated hydrocarbons by force-field method. J. Am. Chem. Soc., 99, 975-986.

Leon-Leon, C.A., Radovic, L.R., 1993. Interfacial Chemistry and Electrochemistry of carbon surfaces. In: Thrower P.A., (Ed.), Chemistry and Physics of Carbon, Vol. 24, Marcel \& Dekker, New York, pp. 213-311.

Lozano-Castello, D., Cazorla-Amoros, D., Linares-Solano, A., 2004. Usefulness of $\mathrm{CO}_{2}$ adsorption at $273 \mathrm{~K}$ for the characterization of porous carbons. Carbon 42, 1233 1242.

Mastral, A.M., García, T., Murillo, R., Callén, M.S., López, J.M., Navarro, M.V., 2003. Measurements of polycyclic aromatic hydrocarbon adsorption on activated carbons at very low concentrations. Ind. Eng. Chem. Res. 42, 155-161.

Moreno-Castilla, C., Rivera-Utrilla, J., Joly, J.P., López-Ramón, M.V., Ferro-García, M.A., Carrasco-Marín, F., 1995. Thermal regeneration of an activated carbon exhausted with different substituted phenols. Carbon 33, 1417-1423.

Olivier, J.P., 1995. Modelling physical adsorption on porous and nonporous solids using density functional theory. J. Porous Mater. 2, 9-17. 
Seredych, M., Gierak, A., 2004. Influence of water on adsorption of organic compounds from its aqueous solutions on surface of synthetic active carbons. Colloids and Surfaces A: Physicochem. Eng. Aspects 245, 61-67.

Seredych, M., Gun'ko, V.M., Gierak, A., 2005. Structural and energetic heterogeneities and adsorptive properties of synthetic carbon adsorbents. Appl. Surf. Sci. 242, 154161.

Terzyk, A.P., 2003. Further insights into the role of carbon surface functionalities in the mechanism of phenol adsorption. J. Colloid Interf. Sci. 268, 301-329.

World Bank Group, 1998. Pollution Prevention and Abatement: Coke Manufacturing. Draft Technical Background Document. Washington, D.C., 1998, p. 286. 


\section{Captions to Tables}

\section{Table 1.}

Chemical composition, PZC, and amounts of $\mathrm{CO}$ and $\mathrm{CO}_{2}$ evolved after heating up to $1000{ }^{\circ} \mathrm{C}$ in $\mathrm{Ar}$ flow

\section{Table 2.}

Textural parameters of the series of carbons studied before and after (sat) the adsorption of naphthalene from aqueous solutions

\section{Table 3.}

Langmuir-Freundlich isotherm parameters for naphthalene removal 


\section{Captions to Figures}

\section{Figure 1.}

Nitrogen adsorption isotherms at $-196{ }^{\circ} \mathrm{C}$ of the carbons studied before and after (sat) naphthalene adsorption.

\section{Figure 2.}

Adsorption isotherms for naphthalene at room temperature on the activated carbons studied. The symbols represent experimental data and the solid lines the fitting to the LF equation.

\section{Figure 3.}

DTG under argon of the samples before and after (sat) naphthalene adsorption.

\section{Figure 4.}

Naphthalene evolution profiles followed by TG-FTIR during the DTG experiments of the samples after naphthalene adsorption.

\section{Figure 5.}

PSD obtained from $N_{2}$ adsorption isotherms at $-196{ }^{\circ} \mathrm{C}$ on the carbons before and after (sat) naphthalene adsorption.

\section{Figure 6.}

$\mathrm{CO}_{2}$ adsorption isotherms of the carbons at $0^{\circ} \mathrm{C}$ before and after (sat) naphthalene adsorption. 
Table 1. Chemical composition, PZC, and amounts of $\mathrm{CO}$ and $\mathrm{CO}_{2}$ evolved after heating up to $1000{ }^{\circ} \mathrm{C}$ in Ar flow

\begin{tabular}{|c|c|c|c|c|c|c|c|}
\hline \multicolumn{5}{|c|}{ Ultimate analysis [wt \%, daf] } & \multirow{2}{*}{ PZC } & \multirow{2}{*}{$\begin{array}{c}\text { CO } \\
{\left[\mu \mathrm{mol} \mathrm{g}^{-1}\right]}\end{array}$} & \multirow{2}{*}{$\begin{array}{c}\mathrm{CO}_{2} \\
{\left[\mu \mathrm{mol} \mathrm{g}^{-1}\right]}\end{array}$} \\
\hline & $\mathbf{C}$ & $\mathbf{H}$ & $\mathbf{N}$ & $\mathbf{O}$ & & & \\
\hline $\mathbf{B}$ & 96.8 & 0.6 & 0.7 & 2.0 & 9.0 & 364 & 122 \\
\hline BS & 86.4 & 0.5 & 0.5 & 12.5 & 2.4 & 2367 & 688 \\
\hline BN20 & 86.9 & 0.5 & 1.3 & 11.3 & 3.5 & 2117 & 837 \\
\hline BN60 & 79.7 & 0.6 & 1.1 & 18.6 & 2.4 & 3216 & 1579 \\
\hline
\end{tabular}

Table 2. Textural parameters of the series of carbons studied before and after (sat) the adsorption of naphthalene from aqueous solutions

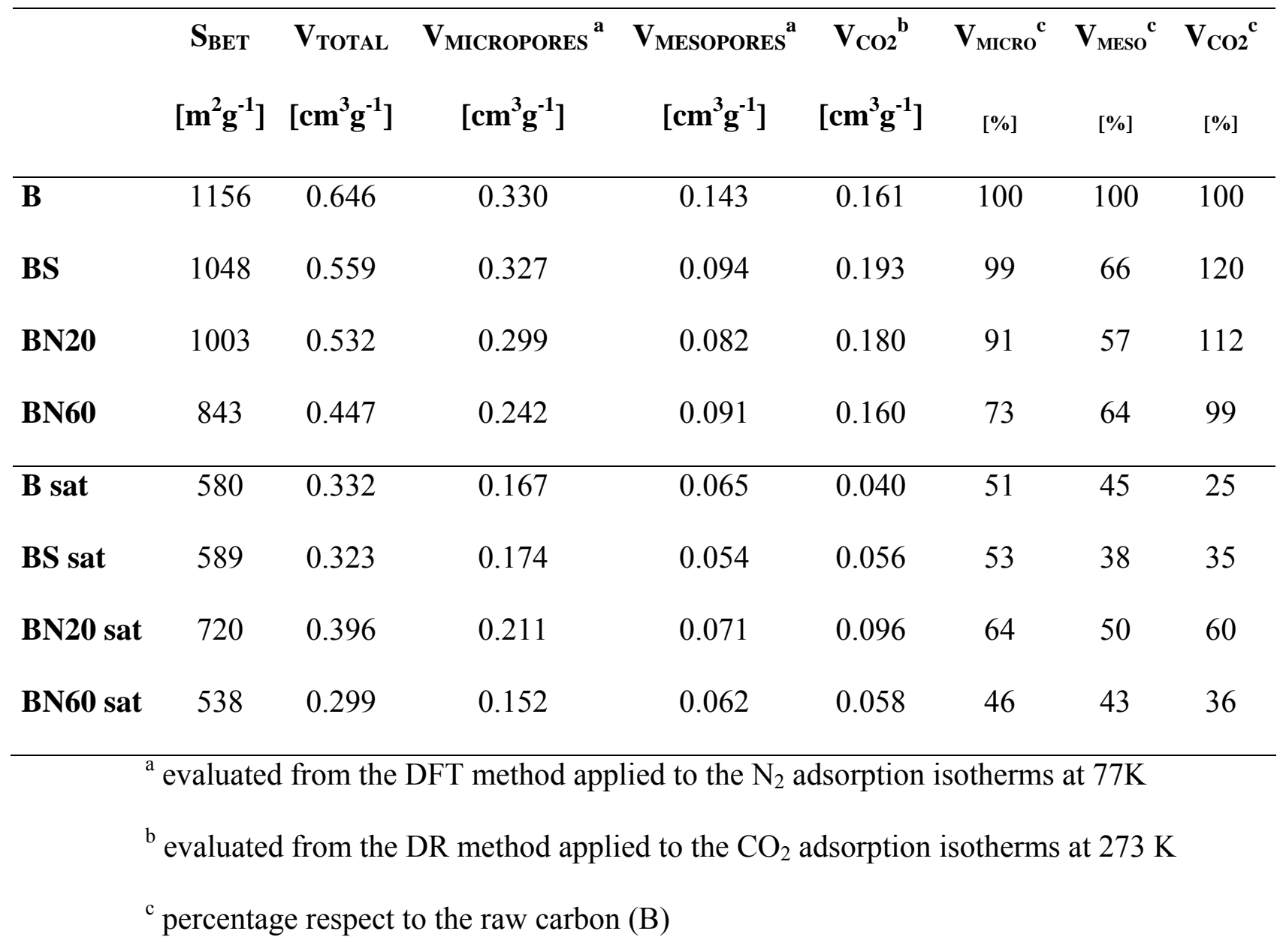


Table 3. Langmuir-Freundlich isotherm parameters for naphthalene removal

\begin{tabular}{lcccc}
\hline & $\begin{array}{c}\text { qm } \\
\end{array}$ & $\mathbf{n}$ & Fitted range & Coefficient $\mathbf{R}^{2}$ \\
& {$\left[\mathbf{m g ~ g}^{-1}\right]$} & & & \\
\hline B & 563 & 0.6 & $5-25 \mathrm{ppm}$ & 0.976 \\
BS & 378 & 0.7 & $0-25 \mathrm{ppm}$ & 0.990 \\
BN20 & 382 & 0.7 & $0-25 \mathrm{ppm}$ & 0.993 \\
BN60 & 185 & 1 & $0-20 \mathrm{ppm}$ & 0.977 \\
\hline
\end{tabular}


Figure 1. Nitrogen adsorption isotherms at $-196^{\circ} \mathrm{C}$ of the carbons studied before and after (sat) naphthalene adsorption.

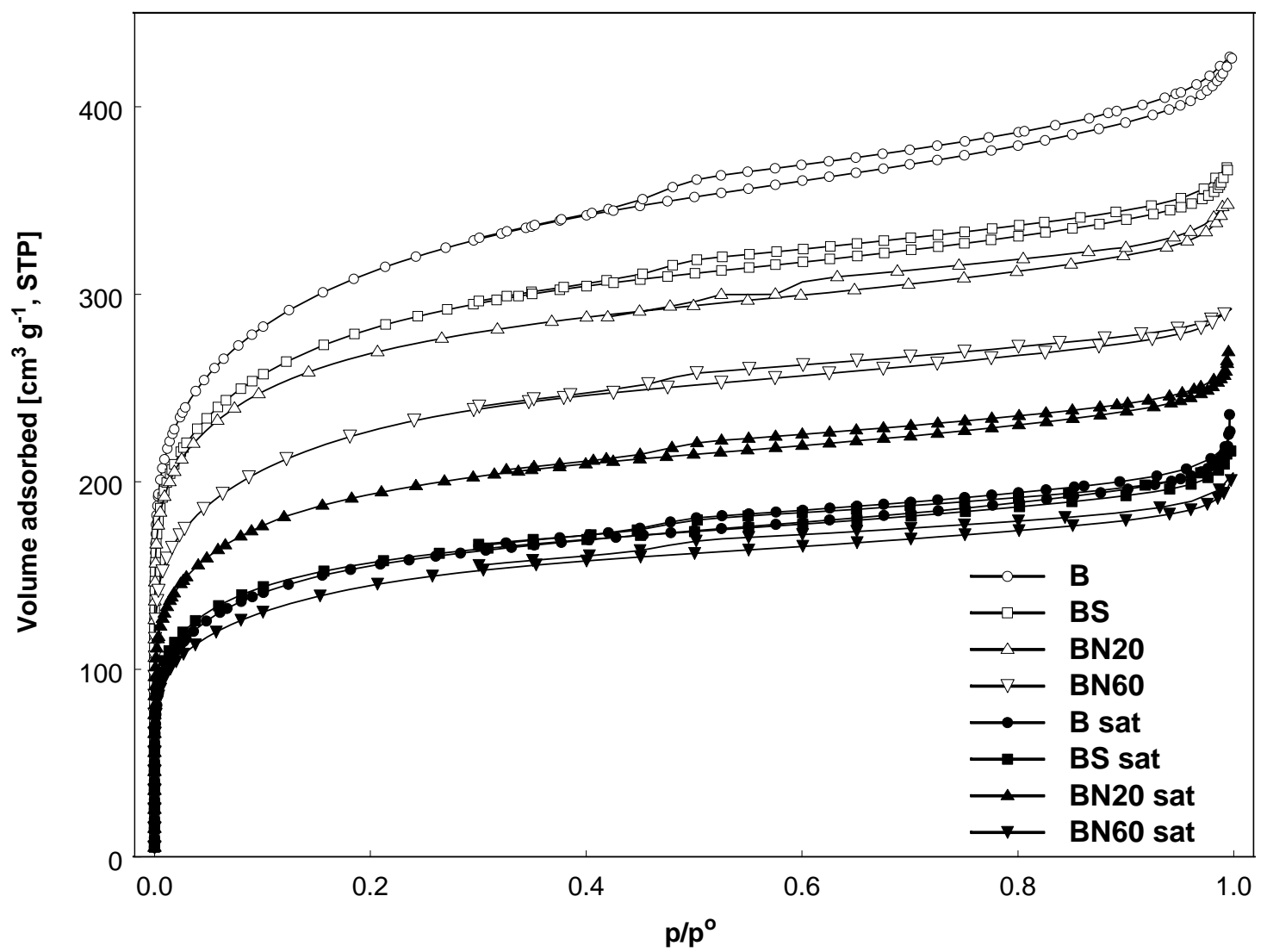


Figure 2. Adsorption isotherms for naphthalene at room temperature on the activated carbons studied. The symbols represent experimental data and the solid lines the fitting to the LF equation.

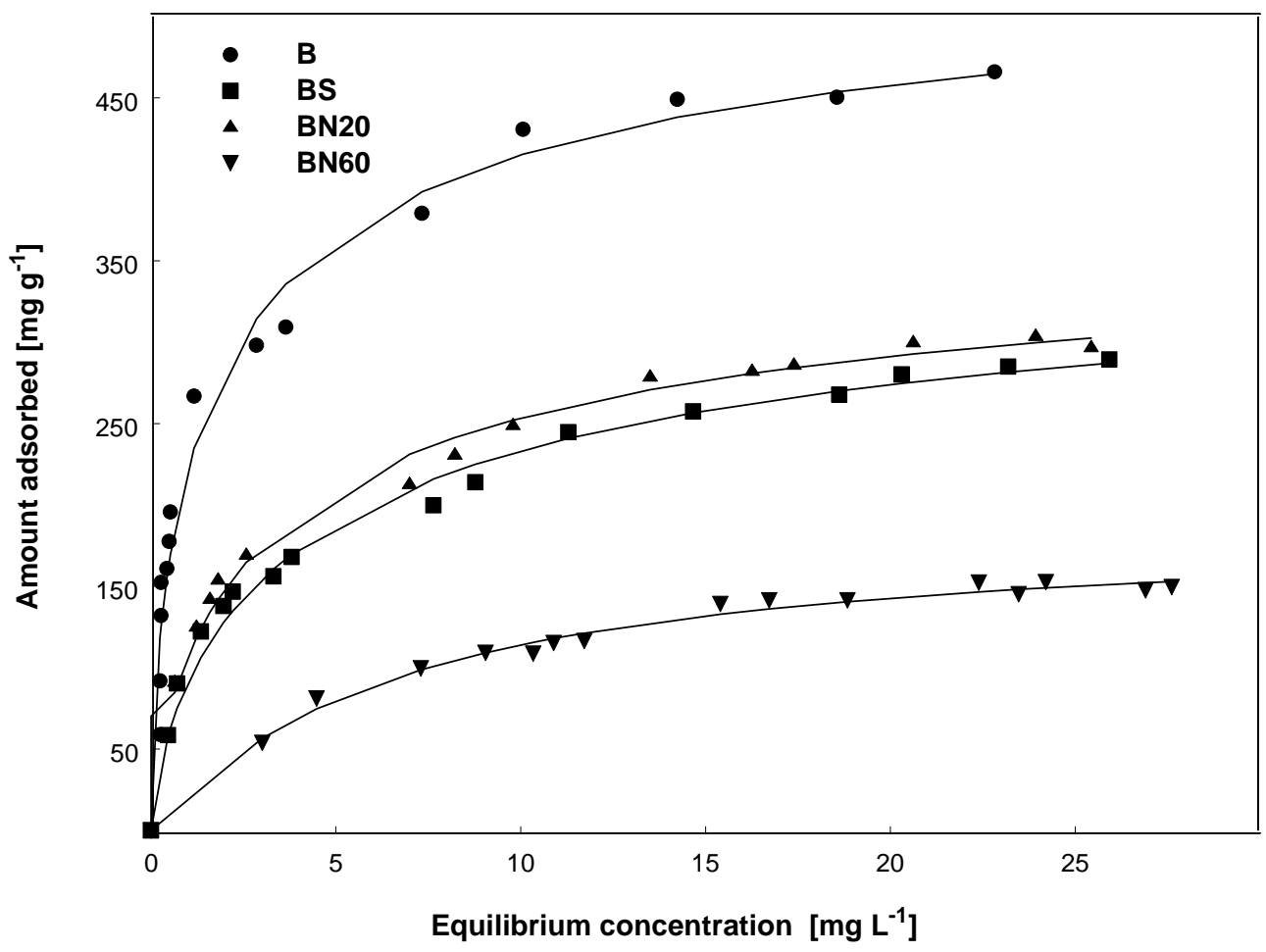


Figure 3. DTG under argon of the samples before and after (sat) naphthalene adsorption.
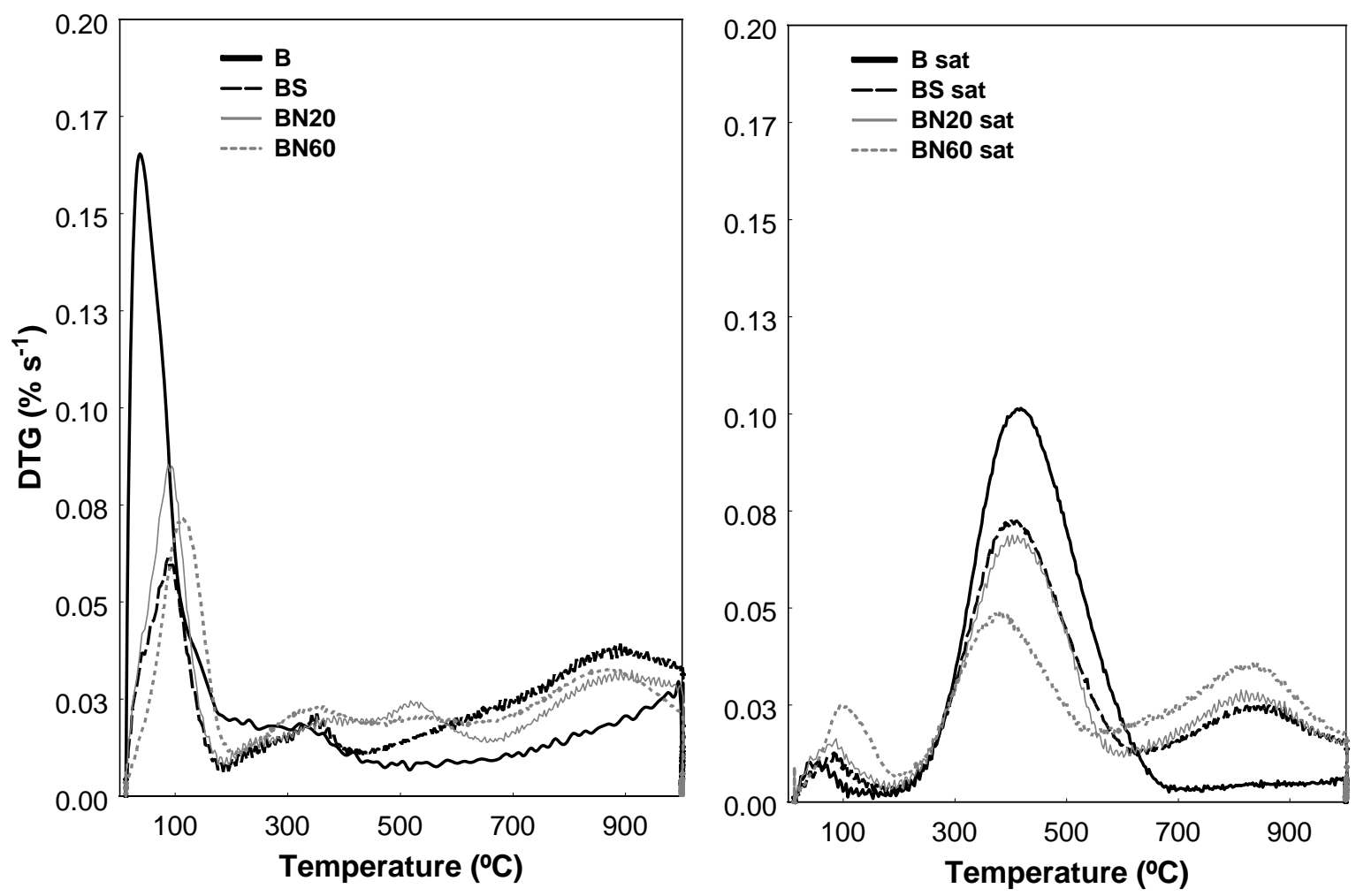
Figure 4. Naphthalene evolution profiles followed by TG-FTIR during the DTG experiments of the samples after naphthalene adsorption.

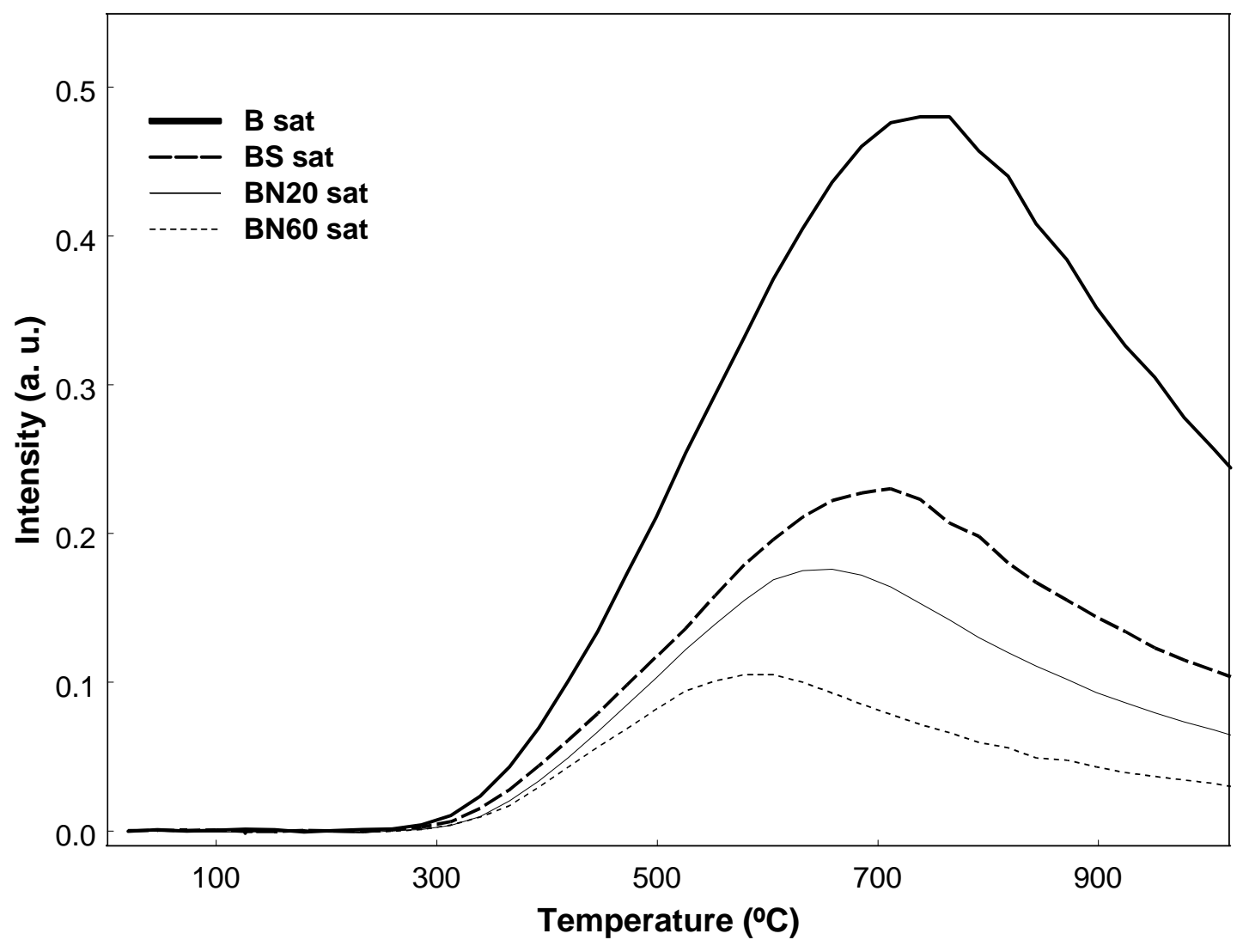


Figure 5. PSD obtained from $\mathrm{N}_{2}$ adsorption isotherms at $-196^{\circ} \mathrm{C}$ on the carbons before and after (sat) naphthalene adsorption.

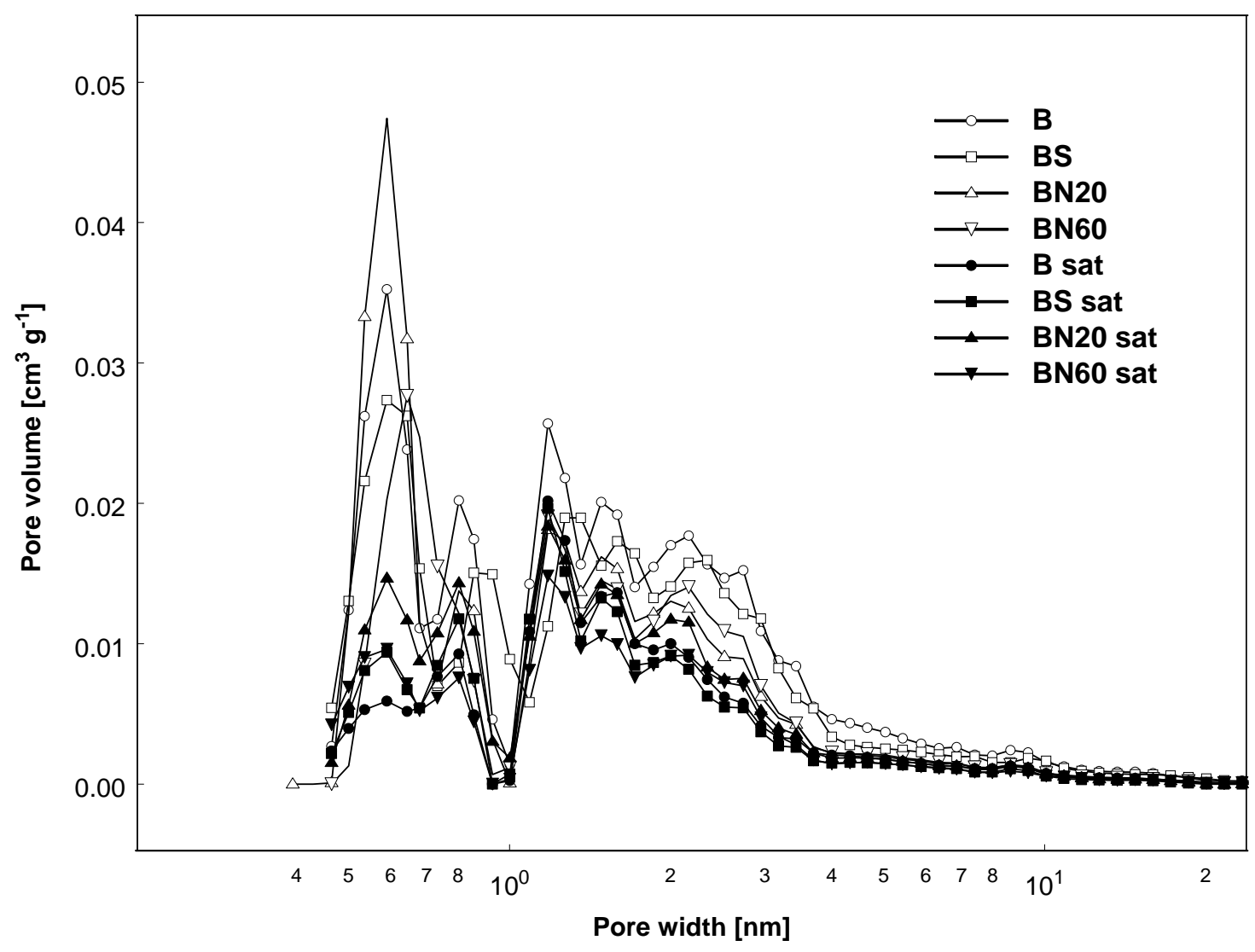


Figure 6. $\mathrm{CO}_{2}$ adsorption isotherms of the carbons at ${ }^{\circ} \mathrm{C}$ before and after (sat) naphthalene adsorption.

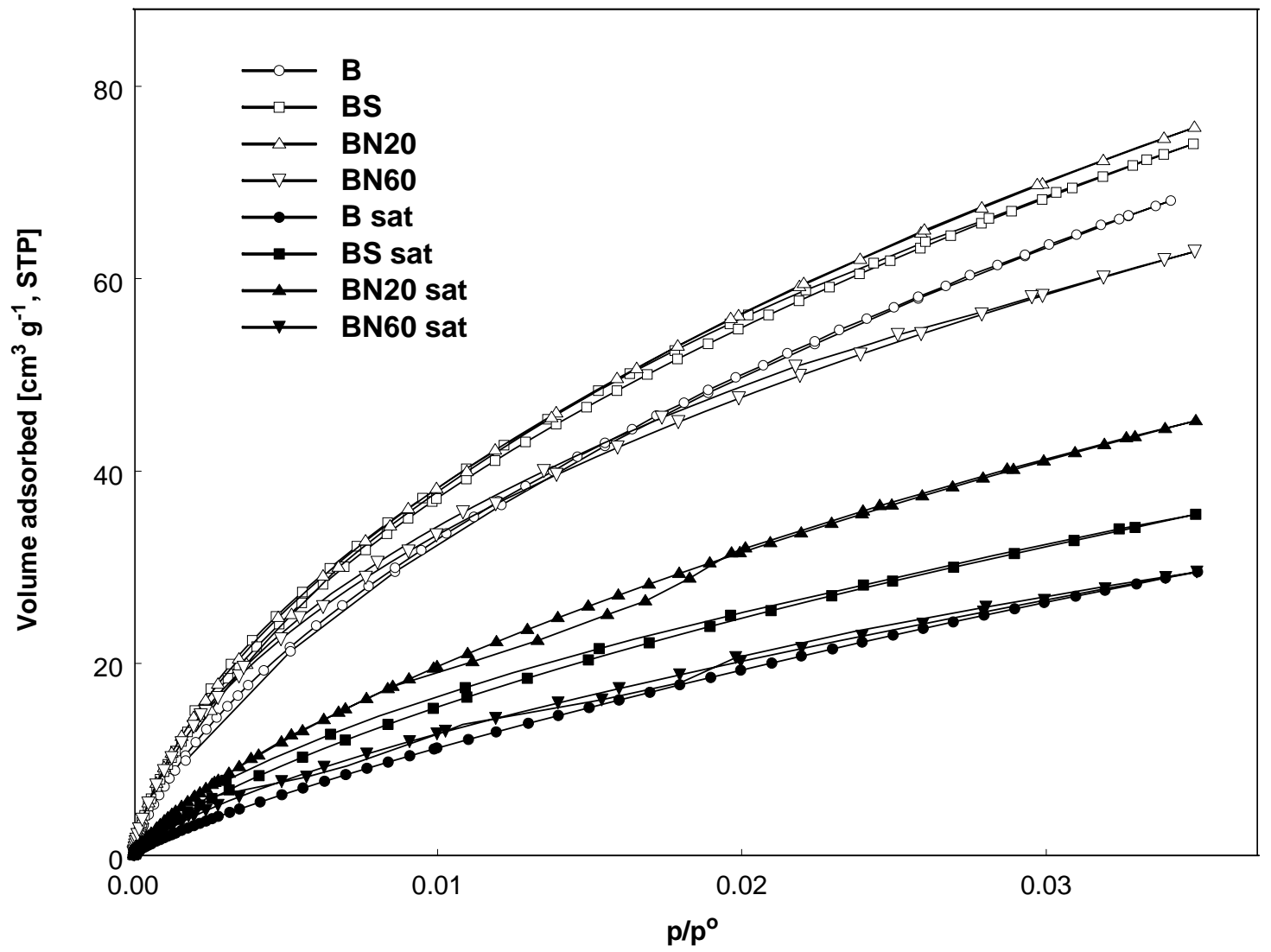

